Velina S. Chavarro, AM*

Maureen A. Mealy, RN, MSCN*

Alexandra Simpson, AM

Anna Lacheta

Florence Pache, MD

Klemens Ruprecht, MD

Stefan M. Gold, PhD

Friedemann Paul, MD

Alexander Ulrich Brandt, $\mathrm{MD} \ddagger$

Michael Levy, MD $\ddagger$

Correspondence to

Dr. Paul:

friedemann.paul@charite.de

Supplemental data at Neurology.org/nn

\section{Insufficient treatment of severe depression in neuromyelitis optica spectrum disorder} OPEN

\section{ABSTRACT}

Objective: To investigate depression frequency, severity, current treatment, and interactions with somatic symptoms among patients with neuromyelitis optica spectrum disorder (NMOSD).

Methods: In this dual-center observational study, we included 71 patients diagnosed with NMOSD according to the International Panel for NMO Diagnosis 2015 criteria. The Beck Depression Inventory (BDI) was classified into severe, moderate, or minimal/no depressive state category. We used the Fatigue Severity Scale to evaluate fatigue. Scores from the Brief Pain Inventory and the PainDETECT Questionnaire were normalized to estimate neuropathic pain. Psychotropic, pain, and immunosuppressant medications were tabulated by established classes.

Results: Twenty-eight percent of patients with NMOSD $(n=20)$ had BDI scores indicative of moderate or severe depression; $48 \%$ of patients $(n=34)$ endorsed significant levels of neuropathic pain. Severity of depression was moderately associated with neuropathic pain $(r=0.341$, $p<0.004$ ) but this relationship was confounded by levels of fatigue. Furthermore, only $40 \%$ of patients with moderate or severe depressive symptoms received antidepressant medical treatment. Fifty percent of those treated reported persistent moderate to severe depressive symptoms under treatment.

Conclusions: Moderate and severe depression in patients with NMOSD is associated with neuropathic pain and fatigue and is insufficiently treated. These results are consistent across 2 research centers and continents. Future research needs to address how depression can be effectively managed and treated in NMOSD. Neurol Neuroimmunol Neuroinflamm 2016;3:e286; doi: $10.1212 / \mathrm{NXI} .0000000000000286$

\section{GLOSSARY}

AQP4 = aquaporin-4; BDI = Beck Depression Inventory; BPI = Brief Pain Inventory; EDSS = Expanded Disability Status Scale; FSS = Fatigue Severity Scale; JHU = Johns Hopkins Hospital; NMO = neuromyelitis optica; NMOSD = neuromyelitis optica spectrum disorder; ON = optic neuritis; PDQ = PainDETECT Questionnaire; SNRI = serotonin norepinephrine reuptake inhibitor; SSRI = selective serotonin reuptake inhibitor; $\mathbf{T M}=$ transverse myelitis.

Depression is one of the most common and debilitating comorbidities of neurologic diseases. It has been associated with decreased quality of life, lower treatment adherence rate, increased disease severity, higher unemployment rates, and cognitive deficits among neurology patients. ${ }^{1}$ Depression is also exacerbated by symptoms of fatigue ${ }^{2}$ and pain, ${ }^{3}$ the interaction of which has a direct effect on quality of life.

Neuromyelitis optica (NMO) spectrum disorder (NMOSD) is an autoimmune disease of the CNS associated with serum antibodies to the astrocyte water channel aquaporin-4 (AQP4). ${ }^{4}$

\footnotetext{
*Equally contributing first authors in alphabetical order.

‡Equally contributing senior authors in alphabetical order.

From NeuroCure Clinical Research Center (V.S.C., A.L., F. Pache, F. Paul, A.U.B.), Clinical and Experimental Multiple Sclerosis Research Center, Department of Neurology (F. Pache, K.R., F. Paul), and Department of Psychiatry and Psychotherapy, Campus Benjamin Franklin (S.M.G.), Charité-Universitätsmedizin Berlin, Germany; Department of Neurology (M.A.M., A.S., M.L.), Johns Hopkins University, Baltimore, MD; Experimental and Clinical Research Center (F. Paul), Max Delbrueck Center for Molecular Medicine, Berlin; and Institute of Neuroimmunology and Multiple Sclerosis (INIMS) (S.M.G.), Center for Molecular Neurobiology, University Medical Center Hamburg-Eppendorf, Germany.

Funding information and disclosures are provided at the end of the article. Go to Neurology.org/nn for full disclosure forms. The Article Processing Charge was paid by the Charité-Universitätsmedizin Berlin Finanzen- und Rechnungswesen/Zentraler Rechnungseingang/Hindenburgdamm 30 12200 Berlin/Vermerk: IA89829082/AG Paul.

This is an open access article distributed under the terms of the Creative Commons Attribution-NonCommercial-NoDerivatives License 4.0 (CC BY-NC-ND), which permits downloading and sharing the work provided it is properly cited. The work cannot be changed in any way or used commercially.
} 
NMOSD is predominantly characterized by optic neuritis and transverse myelitis leading to blindness, weakness, numbness, and pain. ${ }^{5}$ Brain areas typically associated with organic depression, ${ }^{6,7}$ such as prefrontal areas, limbic systems, and the hippocampus, are usually spared among patients with NMOSD. ${ }^{8-10}$ However, recent studies have reported a high prevalence of depression within this population and have claimed that patients with NMOSD present with depression severity comparable to that experienced by patients with multiple sclerosis. ${ }^{1-13}$ Due to the low prevalence of NMOSD, ${ }^{14,15}$ previous studies have employed smaller samples and international comparisons are currently missing. Therefore, it is imperative to investigate the elements of depression among patients with NMOSD within a larger multicenter cohort.

We assessed the frequency and severity of depressive states and their interaction with somatic symptoms in a multicenter observational investigation across 2 continents. Our secondary objective was to evaluate the association of disease-modifying, pain, and psychotropic therapy with current depressive and somatic symptoms.

METHODS Sample. We collected data in a cross-sectional observational study at 2 major academic research centers: the Charité Universitätsmedizin, Berlin, Germany (the Charité) and the Johns Hopkins Hospital (JHU), Baltimore, MD.

Inclusion criteria were formal diagnosis of NMOSD according to the International Panel for NMO Diagnosis 2015 updated diagnostic criteria ${ }^{16}$ and conversational level of the primary language spoken at the recruiting center. Exclusion criteria were age over 70 years and myelin oligodendrocyte glycoprotein antibody seropositivity when available. ${ }^{17}$ All patients at each center who satisfied the inclusion criteria and did not meet the exclusion criteria were included in this study.

Standard protocol approvals, registrations, and patient consents. Study initiation followed research protocol approval by the internal ethics review board at each institution. Patients provided written informed consent and underwent a formal neurologic examination.

Assessment of depressive symptomatology. To evaluate depressive symptoms, we administered the Beck Depression Inventory (BDI)-IA ${ }^{18}$ at the Charité and BDI-II version ${ }^{19}$ at JHU. Both versions contain 21 questions with a maximum total score of 63. Total BDI-IA scores were adjusted to BDI-II scores according to the BDI-II Manual. ${ }^{20}$ We interpreted BDI scores as follows: 0-9 as nondepressive affect; $10-19$ as minimal mood disturbance; 20-29 as moderate depressive state; 30 and above as severe depressive state. ${ }^{20}$ Consistent with prior literature, we categorized patients whose total BDI score was $\geq 20$ with a high likelihood of clinical depression..$^{21,22}$
Assessment of fatigue. We assessed fatigue with the Fatigue Severity Scale (FSS). ${ }^{23}$ This questionnaire measures severity of fatigue in 9 questions on a 7-point Likert scale. A threshold level of 4 has been shown to differentiate individuals with and without significant levels of fatigue. ${ }^{2,23,24}$

Neuropathic pain. The Brief Pain Inventory (BPI) and the PainDETECT Questionnaire (PDQ) were used to evaluate presence and severity of pain at JHU and the Charité, respectively.

The BPI was created to measure pain level from 0 (least) to 10 (worst) in cancer patients in the United States. ${ }^{25}$ Among patients with nonmalignant chronic pain, an average BPI score of 6.8 (SD 1.8) has been reported ${ }^{26}$ and recent work has shown that patients with NMO average a BPI score of 5.9 (SD 2.9). ${ }^{27}$ Based on these findings, we adopted a conservative cutoff score of 6 : patients who scored $<6$ on the BPI index were classified as not having pain with a significant neuropathic component while those scoring $\geq 6$ were classified as experiencing neuropathic pain. Although a conservative cutoff score of BPI $=6$ may have decreased sensitivity of this measure, it minimized overestimating the prevalence of neuropathic pain.

The PDQ was created to differentiate nociceptive from neuropathic pain in low back pain patients. ${ }^{28}$ According to validation data representative of the German population, ${ }^{26}$ a PDQ score of 12 or higher is indicative of pain with a primarily neuropathic etiology. We classified individuals with scores $\geq 12$ as experiencing neuropathic pain, and those with scores $<12$ as not experiencing pain with a substantial neuropathic component.

To allow for a quantitative comparison of pain within the entire cohort, we tested the distribution of BPI and PDQ scores for normality with Kolmogorov-Smirnov tests and standardized individual values to the mean of their respective recruiting center sample. After pooling, $z$ scores of pain levels used in all statistical tests ranged from -1.68 to 2.085 (mean -0.0001 , SD 0.993), representing average and $\mathrm{SD}$ of pain within our cohort.

Current pharmacotherapy. We recorded immunosuppressive preventive therapy for NMOSD, including rituximab, azathioprine, mycophenolate, and methotrexate. ${ }^{29,30}$ Pain medications included anticonvulsants (gabapentin, pregabalin, carbamazepine, clonazepam, oxcarbazepine, topiramate, zonisamide), muscle relaxants (baclofen, tizanidine, diazepam, dantrolene, tolperisone), tricyclic antidepressants (amitriptyline), opioids (tramadol, fentanyl, oxycodone), and a selective serotonin norepinephrine reuptake inhibitor (SNRI; duloxetine). The nature and etiology of pain reported by patients with NMOSD is often managed with multiple medication categories, most frequently with anticonvulsants and low-dose tricyclic antidepressants. ${ }^{31-33}$ Psychoactive medications included SNRI (duloxetine, venlafaxine, milnacipran), selective serotonin reuptake inhibitors (SSRI; citalopram, escitalopram, sertraline), tricyclic antidepressants (amitriptyline), and atypical antidepressants (mirtazapine).

Statistical analyses. We examined the demographic and clinical characteristics of patients with BDI $\geq 20$ and those with BDI $<20$. Continuous variables between groups were compared with independent samples $t$ tests; categorical variables, with Pearson $\chi^{2}$ tests; effect sizes are reported with Cohen $d$. Distributions of all outcome variables were assessed for normality with Kolmogorov-Smirnov tests. Nonparametric tests were used to assess non-normally distributed continuous outcomes, ordinal variables, and the effect of treatment on the primary outcomes. We performed Pearson correlations of primary outcomes and partial correlations adjusting for confounders. We utilized a stepwise multiple regression to evaluate the variability of BDI scores attributable to somatic symptoms 
and clinical and demographic factors. All tests were 2-tailed and significance was indicated when $p<0.05$.

RESULTS Demographics. Seventy-one patients with NMOSD ( $\mathrm{n}=36$ at JHU, $\mathrm{n}=35$ in Berlin), $89 \%$ of whom were AQP4-positive, were enrolled in this study. Ninety-three percent of all participants were female; the female to male ratio was typical of relapsing NMOSD. ${ }^{9}$ The mean disease duration was 7.1 years (SD 5.6) and the median Expanded Disability Status Scale (EDSS) of the sample was 4.5 (range 0-8). Participants did not differ on demographic and clinical variables between centers, except on ethnic distribution and EDSS. Table 1 illustrates the characteristics of the entire cohort and clinical and demographic characteristics of the sample by recruitment center are shown in table e-1 at Neurology.org/nn.

Frequency of moderate or severe depression. We found that $30 \%(n=21)$ of patients with NMOSD scored between 10 and 19, consistent with a dysphoric state; $22 \%(\mathrm{n}=16)$ scored between 20 and 29, consistent with moderate depressive state; and $6 \%(n=4)$ scored above 30, consistent with severe depression.

$\begin{array}{ll}\text { Table } 1 \quad \text { Participant characteristics } \\ \begin{array}{l}\text { Demographic characteristics } \\ \text { Age, } \mathbf{y}\end{array} \\ \begin{array}{l}\text { Sex, \% } \\ \text { Female }\end{array} \\ \begin{array}{l}\text { Male }=71) \\ \text { Ethnicity, \% }\end{array} \\ \text { European descent } & 93 \\ \text { African descent } & 7 \\ \text { Asian descent } & 75 \\ \text { Clinical characteristics } & 22 \\ \text { AQP4 seropositive+, \% } & 3 \\ \text { EDSS } & 89 \\ \text { Disease duration } & 4.5(0-8) \\ \text { BDI score } & 7.1(5.6) \\ \text { FSS score } & 13.6(9.2) \\ \text { Pain Z score } & 4.4(1.7) \\ \text { PDQ } & 0.0(1.0) \\ \text { BPI } & 12.8(8.7) \\ \end{array}$

Abbreviations: AQP4 = aquaporin-4; BDI = Beck Depression Inventory adjusted total scores; BPI = Brief Pain Inventory; EDSS = Expanded Disability Status Scale; FSS = Fatigue Severity Scale; PDQ = PainDETECT Questionnaire. Pain $Z$ score = normalized BPI and PDQ scores. Age, disease duration, $\mathrm{BDI}, \mathrm{FSS}$, and pain $Z$ scores are reported as mean (SD). EDSS is reported as median (range).
Fifteen patients with NMOSD (21\%) also endorsed suicidal ideation or behavior, indicated by a score of 1 or greater on item 9 of the BDI. Seventy-five percent of patients who reported suicidal ideation or behavior scored 20 or greater and the distribution of suicidal patients did not differ across centers.

Patients with BDI scores $\geq 20$ and patients with BDI scores $<20$ did not differ with regard to age (mean 47.1, SD 11.1 vs mean 45.7, SD 12.2, $p>$ 0.1 ), disease duration (mean 5.7, SD 4.2 vs mean 7.7 , SD 5.3, $p>0.1$ ), or EDSS (median 5.5, 1-7 vs median 4.0, 0-8, $U=421.5, p>0.1)$. Distribution of BDI scores was similar across the 2 recruiting centers. The figure illustrates the severity of depressive symptoms by center (A) and antidepressant treatment (B).

History and relapse rate of optic neuritis (ON), transverse myelitis (TM), or both $\mathrm{ON}$ and TM was available only for the cohort from the Charité. BDI scores between patients with any of the 3 classifications (ON, TM, or ON and TM) did not differ, $F=$ $0.667, p=0.520$. Furthermore, relapse rate was not associated with BDI scores, Pearson $r(26)=0.212$, $p=0.297$. The 6 patients with monophasic disease had an average BDI score mean $=14.5$ (SD 9.1), which was not different from the entire cohort mean.

Depression associated with higher levels of fatigue and neuropathic pain. Patients with moderate to severe depressive states had significantly higher FSS scores than patients with mild or minimal depressive states and experienced neuropathic pain of greater intensity. With the exception of one patient, all who scored 20 or greater on the BDI had elevated FSS scores (table 2). BDI scores were moderately correlated with standardized pain scores, Pearson $r(71)=$ $0.341, p<0.004$. However, when fatigue was controlled for, this association became weaker, Pearson $r(66)=0.140, p=0.254$.

In a multiple regression model, pain and fatigue accounted for $23 \%$ of the variance in depressive states, $R^{2}=0.23, F_{2,66}=9.67, p<0.001$. Demographic or clinical parameters, including EDSS, did not contribute to explaining the variance of depressive states beyond pain and fatigue. Of note, lower disease duration $(\beta=-0.247, p=0.244)$ and greater EDSS ( $\beta=0.772, p=0.271)$ were associated with an increase in BDI score.

Somatic symptoms and prescription medication therapy. Of the 68 participants who provided information on antidepressant medications, a total of 20 patients received antidepressant pharmacotherapy, either as psychiatric or pain therapy. There was no difference in the distribution of antidepressant medications reported by patients across centers, Pearson $\chi^{2}(3,68)=5.896, p=0.117$. Table e-2 
A

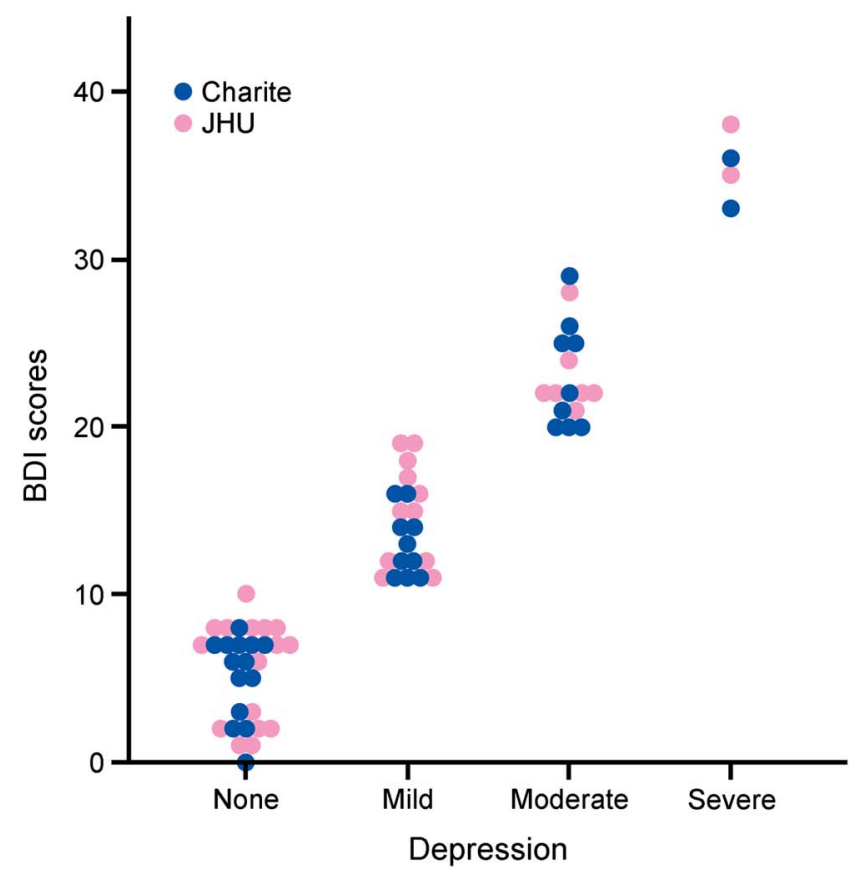

B

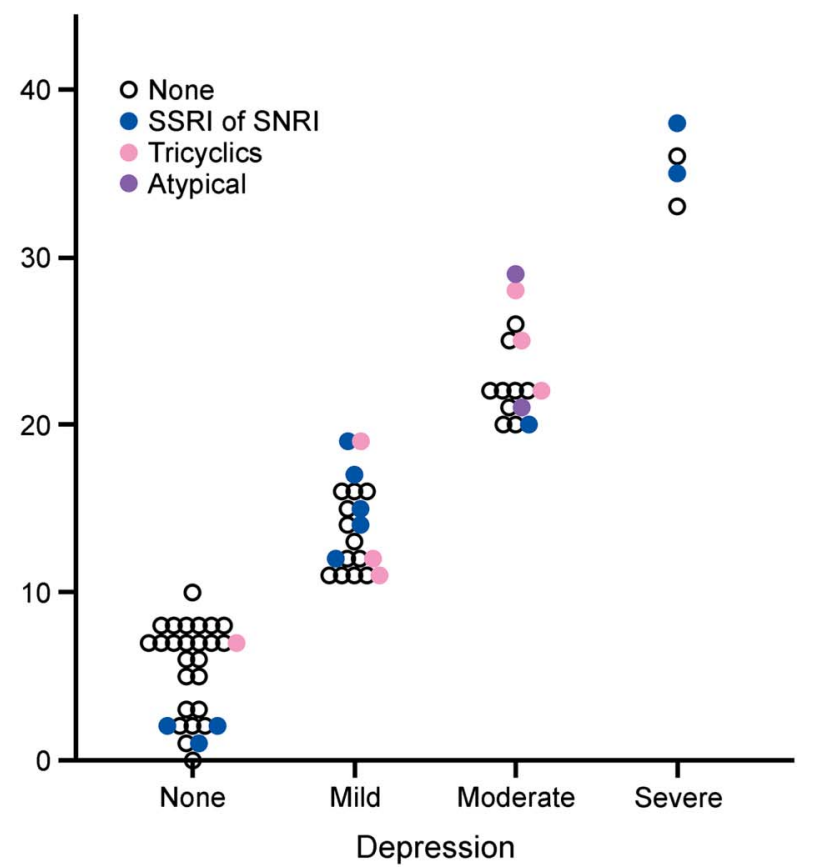

(A) Labeled by recruiting center. (B) Labeled by antidepressant medication therapy. BDI = Beck Depression Inventory; JHU = Johns Hopkins Hospital; SNRI = serotonin norepinephrine reuptake inhibitor; SSRI = selective serotonin reuptake inhibitor.

presents the distribution of medications by center. Fifty percent of those (10 out of 20) still scored 19 or above on the BDI and only 4 scored within the normal range of BDI. Medically treated patients were $40 \%$ (8 out of 20 ) of the total number of patients with BDI score $\geq 20$. Half of the patients categorized as severely depressed ( 2 out of 4 ), based on a BDI score above 30 , received antidepressant medication therapy. A total of 6 out of 16 patients with moderate depression received antidepressant therapy. Among patients who reported moderate depression, antidepression medications included tricyclics $(\mathrm{n}=3)$, atypical antidepressants $(\mathrm{n}=2)$, and

\begin{tabular}{|c|c|c|c|c|}
\hline \multirow{2}{*}{$\begin{array}{l}\text { Table } 2 \\
\text { Syndrome }\end{array}$} & \multicolumn{4}{|c|}{$\begin{array}{l}\text { Prevalence of fatigue and pain in depressed and nondepressed } \\
\text { patients with neuromyelitis optica spectrum disorder }\end{array}$} \\
\hline & $\begin{array}{l}\text { Not depressed, } \\
\text { BDI <20 }\end{array}$ & $\begin{array}{l}\text { Depressed, } \\
\text { BDI } \geq 20\end{array}$ & Test statistic & Effect size \\
\hline \multicolumn{5}{|l|}{ Fatigue } \\
\hline FSS score & 4.05 (1.72) & $5.24(1.47)$ & $t(67)=2.72$ & $d=0.74$ \\
\hline Prevalence & 0.57 & 0.90 & $x^{2}(2)=6.90$ & $V=0.31$ \\
\hline \multicolumn{5}{|c|}{ Neuropathic pain } \\
\hline$Z$ score & $-0.20(0.98)$ & $0.50(0.85)$ & $t(69)=2.78$ & $d=0.76$ \\
\hline Prevalence & 0.41 & 0.65 & $x^{2}(2)=3.27$ & $V=0.22$ \\
\hline
\end{tabular}

Abbreviations: BDI = Beck Depression Inventory adjusted total scores; FSS $=$ Fatigue Severity Scale.

Pain $Z$ score = Brief Pain Inventory and PainDETECT Questionnaire scores normalized to the sample mean. Values are mean (SD) or proportions.
SNRI $(n=1) ; 3$ of these patients were receiving antidepressants for the indication of pain and not depression.

Fifty-two percent of patients received pain medication therapy (36 out of 69), the majority (56\%, 20 out of 36) filling current prescriptions for more than one category of pain medication. Of note, the distribution of pain medications differed across recruiting centers, Pearson $\chi^{2}(3,69)=11.887, p=$ 0.018 (table e-2). Twenty-five percent (9 out of 36) were taking only anticonvulsants, $17 \%$ (6 out of 36 ) were taking only muscle relaxants, and 1 patient was prescribed the opioid/SNRI tramadol. All patients who received low-dose antidepressants as indicated for pain treatment were also receiving other classes of pain medications. Among patients with NMOSD prescribed any pain medications, 58\% (21 out of 36) still endorsed current pain above the threshold for clinically significant neuropathic pain (PDQ $>12$ and BPI $>6$ ).

Patients who receive pain medications and no psychopharmacotherapy have lower depressive states and fatigue. Patients who received only pain therapy scored almost 8 points lower on the total BDI score (mean 10.26, SD 5.32, $\mathrm{n}=19$ ) than those who received both psychotropic and pain medications (mean 18.00, SD 10.78, $\mathrm{n}=16, t[33]=2.614$, $p=0.016, d=0.911)$. Patients who took lowdose antidepressants indicated for pain treatment 
were included in the pain and antidepressants medications group. The category of psychopharmacologic therapy did not have an effect on BDI scores stratified by pain medication. Further, among the 16 patients who received both psychotropic and pain medications, half had a BDI score $>20$, in comparison to only 2 among the 19 patients who received only pain medications.

Patients with NMOSD who received only pain therapy had lower fatigue scores (mean 4.47, SD $1.78, \mathrm{n}=19)$ than patients who received both pain and psychoactive medications (mean 4.60, SD 1.70, $\mathrm{n}=16$ ) and patients who received only psychotropic medications (mean 4.95, SD 1.19, $\mathrm{n}=4$ ). In comparison, patients with NMOSD who received neither pain nor psychoactive medications had an average FSS score mean 4.00, SD 1.70, $\mathrm{n}=27$. This difference in fatigue scores did not reach significance.

Immunosuppressive therapy is differentially associated with depression, fatigue, and pain. When stratified by current immunosuppressant therapy, patients who were treated with rituximab scored lowest on the BDI (mean 11.83, SD 7.51, $\mathrm{n}=41$ ) compared to patients who received mycophenolate (mean 16.27, SD $11.70, \mathrm{n}=11)$ and patients receiving azathioprine (mean 17.13, SD 11.18, $\mathrm{n}=14$ ). A nonparametric test of rank differences did not reach significance. Additionally, across patients who received rituximab, BDI scores were similar regardless of pain or psychotropic medications. Table 3 illustrates pain, fatigue, and depression levels tabulated by immunosuppressant medication.

DISCUSSION In this transnational comparison of depressive symptomatology in NMOSD, moderate to severe depression was observed in $28 \%$ of patients. Our results exhibit a consistency of depressive and somatic symptomatology across 2 research centers and continents. We observed no significant differences in the prevalence or severity of depression, fatigue,

\begin{tabular}{|c|c|c|c|c|c|}
\hline \multirow[t]{3}{*}{ Table 3} & \multicolumn{5}{|c|}{$\begin{array}{l}\text { Beck Depression Inventory adjusted total scores (BDI), Fatigue } \\
\text { Severity Scale (FSS), and Brief Pain Inventory/PainDETECT } \\
\text { Questionnaire scores stratified by immunosuppressant medication }\end{array}$} \\
\hline & & \multicolumn{3}{|c|}{ Immunosuppressant therapy ${ }^{a}$} & \multirow[b]{2}{*}{ p Value ${ }^{b}$} \\
\hline & & $\begin{array}{l}\text { Rituximab } \\
(n=41)\end{array}$ & $\begin{array}{l}\text { Azathioprine } \\
(n=14)\end{array}$ & $\begin{array}{l}\text { Mycophenolate } \\
(n=11)\end{array}$ & \\
\hline BDI score & & $11.83(7.51)$ & $17.36(11.18)$ & $16.27(11.70)$ & 0.242 \\
\hline FSS score & & $4.54(1.67)$ & $4.25(1.78)$ & $3.89(2.18)$ & 0.672 \\
\hline Neuropathi & pain $Z$ score & 0.02 (1.03) & $-0.45(1.07)$ & $0.42(0.72)$ & 0.086 \\
\hline
\end{tabular}

Pain Z score = Brief Pain Inventory and PainDETECT Questionnaire scores normalized to the sample mean.

Values are mean (SD).

a The one patient who was taking methotrexate was excluded from this analysis.

b Significance results from Kruskal-Wallis nonparametric rank test. or pain between patients in Germany and patients in the United States. These findings are in line with recent research from the United Kingdom, France, and Japan. ${ }^{20,22,34}$

As established in cohorts with major depression, suicidality was the most common symptom associated with high scores on the BDI. ${ }^{35,36}$ Prevalence and severity of BDI scores were not associated with history of myelitis or optic neuritis. Further, within our sample, patients with moderate to severe depression had significantly higher levels of pain and fatigue than patients with NMOSD with no or minimal depressive symptoms. Similar findings are also reflected in previous studies largely demonstrating a positive correlation between pain and depressive affect $^{3,37,38}$ as well as between fatigue and depressive affect. $^{39,40, \mathrm{el}}$

Although pain is moderately and significantly correlated with depression among patients with NMOSD, levels of fatigue account for the majority of this association. Interestingly, physical disability or disease duration did not contribute significantly to the variance of depressive states. This provides further evidence for pain as antecedent to depression proposed by Fishbain et al. ${ }^{3}$ Our study design prevents us from showing a causal relationship; however, when stratified by immunosuppressant therapy, we observed that BDI scores are high when levels of pain and fatigue are inversely related. This may indicate that the interaction of fatigue and pain is differentially modulated by immunosuppressant therapy and specifically contributes to depressive mood in this cohort.

We also show that despite receiving antidepressant medications, half of patients with NMOSD still scored 20 or above on the BDI, suggesting insufficient or inadequate management of depressive symptomatology. Interestingly, we observed that if taken concurrently with pain medications, antidepressant medication therapy is associated with significantly higher BDI scores. Although these results must be interpreted with caution due to our small sample sizes, they may suggest that patients who experience only pain, and therefore take only pain medications, report lower depression levels. However, when depressive states are comorbid with pain, the additional burden appears not adequately alleviated despite multiple classes of pharmacotherapy.

Prescriptions of pain medications vary greatly among patients taking different disease-modifying therapies. However, there were no statistically significant differences in depression severity among patients treated with different disease-modifying therapies. The cross-sectional design of our study does not allow us to infer whether immunosuppressant, pain, and depression therapy specifically affect 
depressive symptoms, fatigue, or pain. Future research should address the specifics of these interactions with larger cohorts in longitudinal or randomized case-control studies.

Our study had some limitations. First, we utilized different versions of the BDI in each research center. BDI-IA probes for responses pertaining to the past week and BDI-II probes for responses pertaining to the past 2 weeks. We adjusted the total scores of BDI-IA to those of BDI-II as recommended by Beck et $\mathrm{al}^{20}$ and used only the adjusted BDI scores in our analyses. We selected a conservative cutoff score of 20 to classify individuals as depressed. In line with current research and clinical recommendations, we define depression as a syndrome of affective and psychosomatic symptoms, and do not imply a diagnosis of major depressive disorder. . $2, \mathrm{e} 3$

Another limitation was the use of different scales to measure pain at each center. The PDQ and the BPI are similar as both measure severity and frequency of neuropathic pain. However, the former captures pain within the past 4 weeks, while the latter captures pain within the last 24 hours. This was an inherent weakness in the design of our study, which we addressed by normalizing PDQ and BPI scores to allow for comparison of pain levels within our cohort. Further, we could not control for preexisting depression or other therapies, such as psychotherapy, and did not have information on duration of treatment with antidepressants. All of these important factors would affect the interpretation of our results.

In the current study, we report that $28 \%$ of patients with NMOSD present with moderate to severe depressive symptomatology, which is substantially above the global estimated rate of 5.5\%-5.9\% of depression in the general population ${ }^{\mathrm{e} 3}$ and is comparable to the $23.7 \%$ (confidence interval $17.4 \%$ $30 \%$ ) prevalence rate of depression observed among patients with multiple sclerosis. ${ }^{\text {e4 }}$ Moreover, we observed that the majority of patients who report depressive symptoms indicative of moderate to severe depressive states do not receive appropriate antidepressant pharmacotherapy, suggesting insufficient or inadequate management of depressive symptomatology among patients with NMOSD. We also show that disease-modifying and pain therapy may modulate levels of depressive symptomatology, and suggest that fatigue and pain may confound this interaction. Future prospective and longitudinal studies with larger cohorts are needed to support our results.

\section{AUTHOR CONTRIBUTIONS}

Study concept and design: F. Paul, A.U. Brandt, M. Levy. Acquisition of data: M.A. Mealy, A. Simpson, A. Lacheta, F. Pache. Analysis and interpretation: V.S. Chavarro, M.A. Mealy, F. Paul, A.U. Brandt, M. Levy.
Critical revision of the manuscript for important intellectual content: V.S. Chavarro, M.A. Mealy, A. Simpson, A. Lacheta, F. Pache, K. Ruprecht, S.M. Gold, F. Paul, A.U. Brandt, M. Levy. Study supervision: K. Ruprecht, F. Paul, A.U. Brandt, M. Levy.

\section{STUDY FUNDING}

This work was supported by Deutsche Forschungsgemeinschaft (DFG Exc 257 to F. Paul and V.S.C. and GO1357/5-2 to S.M.G.) and Bundesministerium für Bildung und Forschung (Competence Network Multiple Sclerosis) to S.M.G., F.P., F. Pache, and K.R.

\section{DISCLOSURE}

V. Chavarro received research support from Deutsche Forschungsgemeinschaft. M.A. Mealy received speaker honoraria from Consortium of Multiple Sclerosis Centers. A. Simpson and A. Lacheta report no disclosures. F. Pache received travel funding from Genzyme, Bayer, Biogen Idec, and ECTRIMS. K. Ruprecht served on the scientific advisory board for Sanofi-Aventis/Genzyme, Novartis, and Roche; received travel funding and/or speaker honoraria from Bayer Healthcare, Biogen Idec, Merck Serono, Sanofi-Aventis/Genzyme, Teva, Novartis, and Guthy Jackson Charitable Foundation; is an academic editor for PLoS One; received royalties from Elsevier; and received research support from the German Ministry of Education and Research. S.M. Gold consulted for UCLA and Cedars Sinai Medical Center and received research support from Deutsche Forschungsgemeinschaft. F. Paul served on the scientific advisory board for Novartis and MedImmune; received speaker honoraria and travel funding from Bayer, Novartis, Biogen Idec, Teva, Sanofi-Aventis/Genzyme, Merck Serono, Alexion, Chugai, MedImmune, and Shire; is an academic editor for PLoS One and an associate editor for Neurology ${ }^{\circledR}$ Neuroimmunology \& Neuroinflammation; has consulted for SanofiGenzyme, Biogen Idec, MedImmune, Shire, and Alexion; and received research support from Bayer, Novartis, Biogen Idec, Teva, Sanofi-Aventis/Genzyme, Alexion, Merck Serono, German Research Council, Werth Stiftung of the City of Cologne, German Ministry of Education and Research, Arthur Arnstein Stiftung Berlin, EU FP7 Framework Program, Guthy Jackson Charitable Foundation, and National Multiple Sclerosis Society of the USA. A.U. Brandt served on the scientific advisory board for Biogen; received travel funding and/or speaker honoraria from Novartis, Bayer, Biogen, and Teva; has a patent pending for method and system for optic nerve head shape quantification, perceptive visual computing based postural control analysis, multiple sclerosis biomarker, and perceptive sleep motion analysis; has consulted for Nexus and Motognosis; received research support from Novartis, Biogen Idec, BMWi, and BMBF; and holds stock or stock options in Motognosis. M. Levy served on the scientific advisory board for Asterias, Chugai, and Alexion; is on the editorial board for Multiple Sclerosis and Related Disorders; holds a patent for Aquaporin- 4 sequence that elicits pathogenic $\mathrm{T}$ cell response in animal model of neuromyelitis optica; has consulted for Guidepoint Global, Gerson Lehrman Group, and Cowen Group; and received research support from Viropharma/Shire, Acorda, ApoPharma, Sanofi, Genzyme, Alnylam, Alexion, Terumo BCT, National Institute of Neurological Disorders and Stroke, and Guthy Jackson Charitable Foundation. Go to Neurology.org/nn for full disclosure forms.

Received April 10, 2016. Accepted in final form August 1, 2016.

\section{REFERENCES}

1. Raskind MA. Diagnosis and treatment of depression comorbid with neurologic disorders. Am J Med 2008;121 (11 suppl 2):S28-S37.

2. Bakshi R, Shaikh ZA, Miletich RS, et al. Fatigue in multiple sclerosis and its relationship to depression and neurologic disability. Mult Scler 2000;6:181-185.

3. Fishbain DA, Cutler R, Rosomoff HL, Rosomoff RS. Chronic pain-associated depression: antecedent or consequence of chronic pain? A review. Clin J Pain 1997;13: 116-137.

4. Zekeridou A, Lennon VA, Zamvil SS, Slavin AJ. Aquaporin-4 autoimmunity. Neurol Neuroimmunol Neuroinflammation 2015;2:e110. doi: 10.1212/NXI.0000000000000110. 
5. Jarius S, Wildemann B, Paul F. Neuromyelitis optica: clinical features, immunopathogenesis and treatment. Clin Exp Immunol 2014;176:149-164.

6. Kupfer DJ, Frank E, Phillips ML. Major depressive disorder: new clinical, neurobiological, and treatment perspectives. Lancet 2012;379:1045-1055.

7. Kempton MJ. Structural neuroimaging studies in major depressive disorder. Arch Gen Psychiatry 2011;68:675-690.

8. Kim HJ, Paul F, Lana-Peixoto MA, et al. MRI characteristics of neuromyelitis optica spectrum disorder: an international update. Neurology 2015;84:1165-1173.

9. Jarius S, Ruprecht K, Wildemann B, et al. Contrasting disease patterns in seropositive and seronegative neuromyelitis optica: a multicentre study of 175 patients. J Neuroinflammation 2012;9:14.

10. Pekcevik Y, Orman G, Lee IH, Meally MA, Levy M, Izbudak I. What do we know about brain contrast enhancement patterns in neuromyelitis optica? Clin Imaging 2016;40:573-580.

11. Moore P, Methley A, Pollard C, et al. Cognitive and psychiatric comorbidities in neuromyelitis optica. J Neurol Sci 2016;360:4-9.

12. Chanson JB, Zéphir H, Collongues N, et al. Evaluation of health-related quality of life, fatigue and depression in neuromyelitis optica. Eur J Neurol 2011;18:836-841.

13. Kawahara Y, Ikeda M, Deguchi K, et al. Cognitive and affective assessments of multiple sclerosis (MS) and neuromyelitis optica (NMO) patients utilizing computerized touch panel-type screening tests. Intern Med 2014;53: 2281-2290.

14. Mealy MA, Wingerchuk DM, Greenberg BM, Levy M. Epidemiology of neuromyelitis optica in the United States. Arch Neurol 2012;69:1176-1180.

15. Pandit L, Asgari N, Apiwattanakul M, et al. Demographic and clinical features of neuromyelitis optica: a review. Mult Scler 2015;21:845-853.

16. Wingerchuk DM, Banwell B, Bennett JL, et al. International consensus diagnostic criteria for neuromyelitis optica spectrum disorders. Neurology 2015;85:177-189.

17. Zamvil SS, Slavin AJ. Does MOG Ig-positive AQP4seronegative opticospinal inflammatory disease justify a diagnosis of NMO spectrum disorder? Neurol Neuroimmunol Neuroinflammation 2015;2:e62. doi: 10.1212/ NXI.0000000000000062.

18. Beck A, Steer R. Manual for the Beck Depression Inventory. San Antonio, TX: Psychological Corporation; 1993.

19. Beck A, Steer R, Ball R, Ranieri W. BDI Comparison of Beck Depression Inventories -IA and -II in psychiatric outpatients. J Pers Assess 1996;67:588-597.

20. Beck A, Steer R, Brown G. Beck Depression Inventory Manual, 2nd ed. Bloomington, IN: PsychCorp Pearson Clinical Assessment; 1996.

21. Arnau RC, Meagher MW, Norris MP, Bramson R. Psychometric evaluation of the Beck Depression Inventory-II with primary care medical patients. Health Psychol 2001; 20:112-119.

22. Sprinkle SD, Lurie D, Insko SL, et al. Criterion validity, severity cut scores, and test-retest reliability of the Beck Depression Inventory-II in a university counseling center sample. J Couns Psychol 2002;49:381-385.

23. Krupp L, LaRocca N, Muir-Nash J, et al. The Fatigue Severity Scale: application to patients with multiple sclerosis and systemic lupus erythematosus. Arch Neurol 1989;46:1121-1123.

24. Flachenecker P, Kümpfel T, Kallmann B, et al. Fatigue in multiple sclerosis: a comparison of different rating scales and correlation to clinical parameters. Mult Scler 2002;8: 523-526.

25. Daut R, Cleeland C, Flanery R. Development of the Wisconsin Brief Pain Questionnaire to asses pain in cancer and other diseases. Pain 1983;17:197-210.

26. Tan G, Jensen MP, Thornby JI, Shanti BF. Validation of the Brief Pain Inventory for chronic nonmalignant pain. J Pain 2004;5:133-137.

27. Zhao S, Mutch K, Elsone L, Nurmikko T, Jacob A. Neuropathic pain in neuromyelitis optica affects activities of daily living and quality of life. Mult Scler 2014;20: 1658-1661.

28. Freynhagen R, Baron R, Gockel U, Tölle TR. PainDETECT: a new screening questionnaire to identify neuropathic components in patients with back pain. Curr Med Res Opin 2006; 22:1911-1920.

29. Kimbrough DJ, Fujihara K, Jacob A, et al. Treatment of neuromyelitis optica: review and recommendations. Mult Scler Relat Disord 2012;1:180-187.

30. Trebst C, Jarius S, Berthele A, et al. Update on the diagnosis and treatment of neuromyelitis optica: recommendations of the Neuromyelitis Optica Study Group (NEMOS). J Neurol 2014;261:1-16.

31. Qian P, Lancia S, Alvarez E, Klawiter EC, Cross AH, Naismith RT. Association of neuromyelitis optica with severe and intractable pain. Arch Neurol 2012;69:1482-1487.

32. Kim SM, Go MJ, Sung JJ, Park KS, Lee KW. Painful tonic spasm in neuromyelitis optica. Arch Neurol 2012: 69:1026-1031.

33. O'Connor AB, Dworkin RH. Treatment of neuropathic pain: an overview of recent guidelines. Am J Med 2009; 122:S22-S32.

34. Jones JE, Hermann BP, Barry JJ, Gilliam F, Kanner AM, Meador KJ. Clinical assessment of axis I psychiatric morbidity in chronic epilepsy: a multicenter investigation. J Neuropsychiatry Clin Neurosci 2005;17:172-179.

35. Beck AT, Steer RA, Beck JS, Newman CF. Hopelessness, depression, suicidal ideation, and clinical diagnosis of depression. Suicide Life Threat Behav 1993;23: 139-145.

36. Beck T, Steer RA, Kovacs M, Garrison B. Hopelessness and eventual suicide: a 10-year prospective study of patients hospitalized with suicidal ideation. Am J Psychiatry 1985;142:559-563.

37. Von Korff M, Simon G. The relationship between pain and depression. Br J Psychiatry Suppl 1996: 101-108.

38. Reyes-Gibby CC, Aday LA, Anderson KO, Mendoza TR, Cleeland CS. Pain, depression, and fatigue in communitydwelling adults with and without a history of cancer. J Pain Symptom Manage 2006;32:118-128.

39. Visser MR, Smets EM. Fatigue, depression and quality of life in cancer patients: how are they related? Support Care Cancer 1998;6:101-108.

40. Dalgas U, Stenager E, Jakobsen J, et al. Fatigue, mood and quality of life improve in MS patients after progressive resistance training. Mult Scler 2010;16: 480-490. 


\section{Neurology \\ Neuroimmunology \& Neuroinflammation}

\section{Insufficient treatment of severe depression in neuromyelitis optica spectrum disorder Velina S. Chavarro, Maureen A. Mealy, Alexandra Simpson, et al. \\ Neurol Neuroimmunol Neuroinflamm 2016;3; \\ DOI 10.1212/NXI.0000000000000286}

This information is current as of October 24, 2016

\section{Updated Information \& Services \\ Supplementary Material}

References

Citations

Subspecialty Collections

Permissions \& Licensing

Reprints including high resolution figures, can be found at: http://nn.neurology.org/content/3/6/e286.full.html

Supplementary material can be found at: http://nn.neurology.org/content/suppl/2016/10/24/3.6.e286.DC1

This article cites 37 articles, 2 of which you can access for free at: http://nn.neurology.org/content/3/6/e286.full.html\#\#ref-list-1

This article has been cited by 1 HighWire-hosted articles: http://nn.neurology.org/content/3/6/e286.full.html\#\#otherarticles

This article, along with others on similar topics, appears in the following collection(s):

All Pain

http://nn.neurology.org//cgi/collection/all_pain

Depression

http://nn.neurology.org//cgi/collection/depression

Information about reproducing this article in parts (figures,tables) or in its entirety can be found online at:

http://nn.neurology.org/misc/about.xhtml\#permissions

Information about ordering reprints can be found online: http://nn.neurology.org/misc/addir.xhtml\#reprintsus

Neurol Neuroimmunol Neuroinflamm is an official journal of the American Academy of Neurology.

Published since April 2014, it is an open-access, online-only, continuous publication journal. Copyright (C) 2016 American Academy of Neurology. All rights reserved. Online ISSN: 2332-7812.

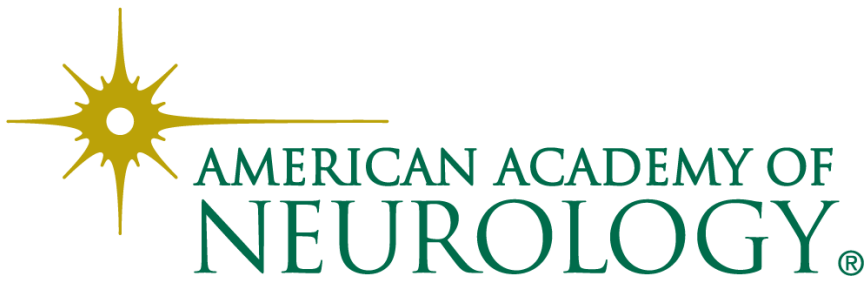

